\title{
Verification of Doppler coherence imaging for 2D ion velocity measurements on DIII- D
}

C. M. Samuell, S. L. Allen, W. H. Meyer, R. C. Isler, A. Briesemeister, R. S. Wilcox, C. J. Lasnier, A. G. Mclean, and J. Howard

Citation: Review of Scientific Instruments 89, 093502 (2018); doi: 10.1063/1.5039367

View online: https://doi.org/10.1063/1.5039367

View Table of Contents: http://aip.scitation.org/toc/rsi/89/9

Published by the American Institute of Physics

\section{Articles you may be interested in}

Diagnostic capacity of electron cyclotron emission imaging system with continuous large observation area on EAST tokamak

Review of Scientific Instruments 89, 093503 (2018); 10.1063/1.5024957

A new type of ionizer for charge-exchange particle analyzer

Review of Scientific Instruments 89, 093504 (2018); 10.1063/1.5043262

An ultrahigh vacuum apparatus for $\mathrm{H}$ atom scattering from surfaces

Review of Scientific Instruments 89, 094101 (2018); 10.1063/1.5047674

A novel, tunable, multimodal microwave system for microwave reflectometry system

Review of Scientific Instruments 89, 093501 (2018); 10.1063/1.5033968

Dual energy-band excitation from a low power Rh anode X-ray tube for the simultaneous determination of low $\mathrm{Z}$ and high $\mathrm{Z}$ elements (Na-U) using total-reflection X-ray fluorescence analysis (TXRF)

Review of Scientific Instruments 89, 093108 (2018); 10.1063/1.5044527

Elliptically bent crystal $x$-ray spectrometer for time-resolved laser plasma experiments

Review of Scientific Instruments 89, 093109 (2018); 10.1063/1.5029462

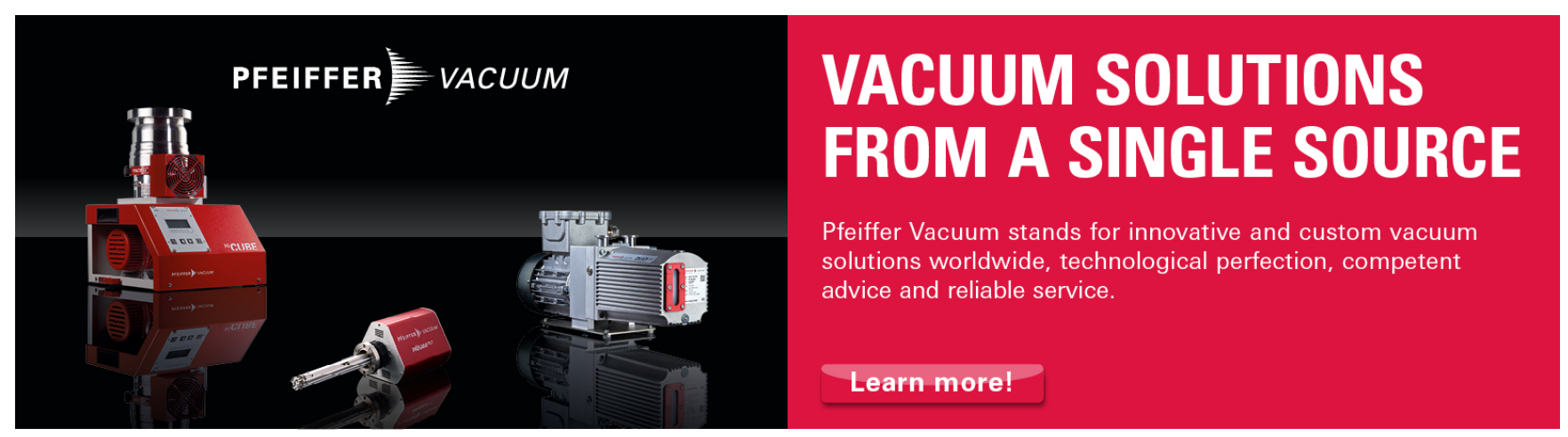




\title{
Verification of Doppler coherence imaging for 2D ion velocity measurements on DIII-D
}

\author{
C. M. Samuell, ${ }^{1, a)}$ S. L. Allen, ${ }^{1}$ W. H. Meyer, ${ }^{1}$ R. C. Isler, ${ }^{2}$ A. Briesemeister, ${ }^{2}$ R. S. Wilcox,${ }^{2}$ \\ C. J. Lasnier, ${ }^{1}$ A. G. Mclean, ${ }^{1}$ and J. Howard ${ }^{3}$ \\ ${ }^{1}$ Lawrence Livermore National Laboratory, Livermore, California 94550, USA \\ ${ }^{2}$ Oak Ridge National Laboratory, Oak Ridge, Tennessee 37830, USA \\ ${ }^{3}$ Australian National University, Canberra, ACT 0200, Australia
}

(Received 7 May 2018; accepted 17 August 2018; published online 18 September 2018)

\begin{abstract}
Coherence Imaging Spectroscopy (CIS) has emerged as a powerful tool for investigating complex ion phenomena in the boundary of magnetically confined plasma devices. The combination of Fouriertransform interferometry and high-resolution fast-framing cameras has made it possible to make sensitive velocity measurements that are also spatially resolved. However, this sensitivity makes the diagnostic vulnerable to environmental effects including thermal drifts, vibration, and magnetic fields that can influence the velocity measurement. Additionally, the ability to provide an absolute calibration for these geometries can be impacted by differences in the light-collection geometry between the plasma and reference light source, spectral impurities, and the presence of thin-films on in-vessel optics. This paper discusses the mitigation of these effects and demonstration that environmental effects result in less than $0.5 \mathrm{~km} / \mathrm{s}$ error on the DIII-D CIS systems. A diagnostic comparison is used to demonstrate agreement between CIS and traditional spectroscopy once tomographic artifacts are accounted for. Published by AIP Publishing. https://doi.org/10.1063/1.5039367
\end{abstract}

\section{INTRODUCTION}

Ion transport along magnetic field lines (flows) drives the transport of particles, energy, and momentum throughout the edge of magnetic fusion devices. Understanding these phenomena, particularly in high powered plasmas, is a prerequisite for the prediction of impurity migration and divertor performance required for the realization of an electricity producing magnetically confined fusion reactor. Measuring the ion velocity of both main-ion and impurity populations is being used to understand the forces that drive these flows in partnership with fluid modeling codes such as $\mathrm{UEDGE}^{1}$ and SOLPS, ${ }^{2}$ as well as fully kinetic simulations. ${ }^{3}$ Significant progress has been made in understanding these complex phenomena using Mach probes, ${ }^{4-7}$ sequential charge-state imaging, ${ }^{1}$ and traditional spectroscopy. ${ }^{8}$ However, these diagnostic techniques become significantly more challenging in high power and high performance scenarios due to the interpretation challenges of line-of-sight spectroscopy, the power handling ability of physical probes, and the possibility of perturbation from both gas puffs and probes.

Doppler Coherence Imaging Spectroscopy (CIS) overcomes many of these limitations as well as provides 2D spatially resolved measurements. This diagnostic technique combines a spatial heterodyne polarization interferometer with a fast-framing camera to generate images of the Doppler shift of any plasma species emitting in the visible spectrum. The interferometer is composed of a birefringent crystal sandwiched between two polarizers that superimposes a set of interference fringes onto the brightness image of plasma emission that has been isolated to a single species using an interference

a)samuell1@1lnl.gov filter. Spectral information of the emitting species including the Doppler shift and width is encoded onto the interferogram and can be extracted using Fourier demodulation techniques. Comparison of these demodulated quantities with those from a spectral reference image can then be used for calculating absolutely calibrated velocities and temperatures. More details on the diagnostic technique can be found in numerous papers by Howard and others. ${ }^{9-13}$ CIS measurements have been made on a number of plasma devices including DIII-D, ${ }^{10,14}$ the MAST spherical tokamak, ${ }^{12}$ the ASDEX-U tokamak, ${ }^{15}$ the H1 heliac, ${ }^{9,16}$ the WEGA stellarator, ${ }^{25}$ and the MAGPIE linear device. ${ }^{17}$

A distinction of the DIII-D setup is the development of permanently installed CIS diagnostic instruments with the aim of providing reliable velocity measurement throughout extended experimental campaigns. Measurements are primarily made of impurity species such as the bright CIII line at $465 \mathrm{~nm}$ and HeII which is a main-ion species in low temperature regions of helium plasmas. The desire to make quantitative comparisons with modeling predictions necessitates a high accuracy and a trustworthy measurement system. The magnitude of velocities in the scrape off layer (SOL) and divertor is in the range of $0-30 \mathrm{~km} / \mathrm{s}$ which drives a target uncertainty of $\pm 1 \mathrm{~km} / \mathrm{s}$ so that the regions of flow stagnation can be identified. Ion velocities measured for a range of discharge conditions can then be used to better constrain modeling efforts ${ }^{14}$ and investigate complicated phenomena such as the presence of 3D flows.

This paper describes work that has been done to verify the CIS diagnostic technique, as implemented on DIII-D. The two Doppler CIS diagnostics installed on DIII-D are described in Sec. II followed by a discussion on the elimination of environmental influences in Sec. III. While benchtop tests have been used to demonstrate the ability of CIS to make accurate 
absolutely calibrated Doppler shift measurements even when extrapolating comparatively large spectral distances, ${ }^{13}$ in situ plasma measurements must be used to confirm that there are no installation-specific errors. This is achieved with a comparison to traditional spectroscopy presented in Sec. IV.

\section{COHERENCE IMAGING SETUP}

Two distinct tangentially viewing Doppler CIS systems are installed on DIII-D. The "main-SOL" system leverages a wide field-of-view to measure velocities in both the upper and lower divertors as well as the intervening main chamber scrape-off-layer (SOL). To achieve this, a high-resolution $2160 \times 2560$ pco.edge camera is combined with a "periscope" optical train to achieve suitable resolution across the view and without optical fibers. The periscope optics setup is shared with an infrared imaging diagnostic that has been described previously. ${ }^{18}$ The second CIS system uses a faster Phantom v7.3 camera to focus on the lower divertor. The faster frame-rate of this system can be used to separate data affected by Edge Localized Modes (ELMs) and inter-ELM data during high power H-mode and advanced-tokamak discharges. Examples of the raw data from these systems are shown in Fig. 1.

(a) Main SOL

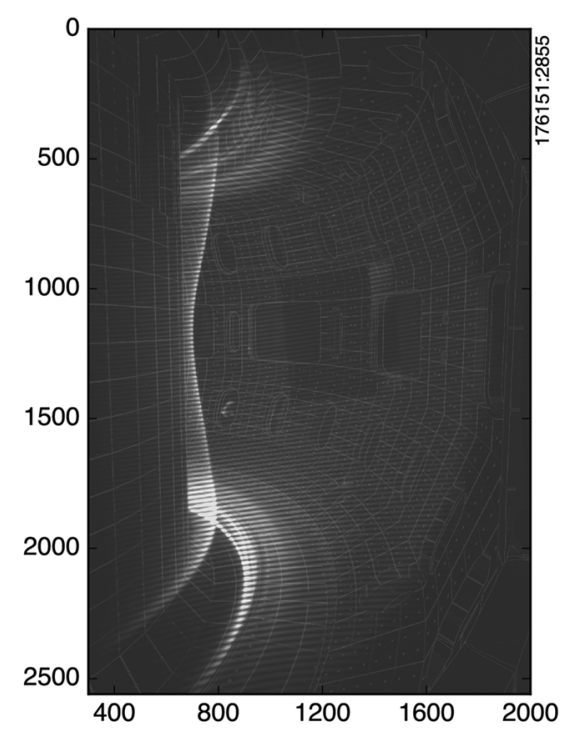

\section{(b) Lower divertor}

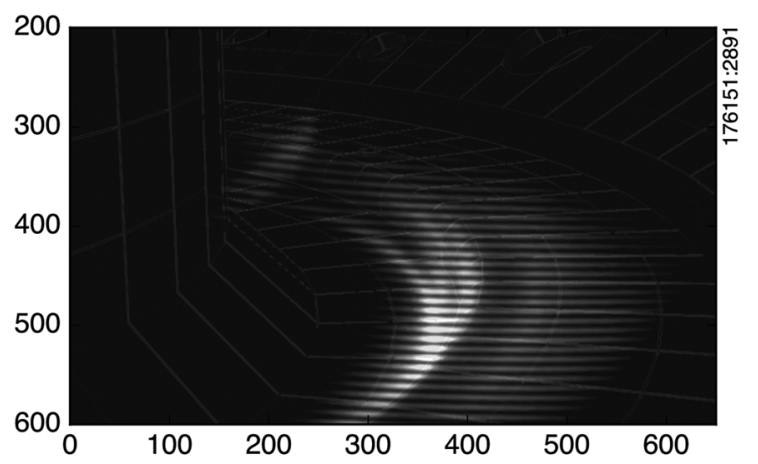

FIG. 1. Raw data $\left(\mathrm{C}^{2+}\right)$ from the (a) main-SOL and (b) lower divertor CIS diagnostics.
Both the main-SOL and lower-divertor systems share a similar design for the interferometer cell which has been detailed elsewhere. ${ }^{13}$ In short, a birefringent crystal is sandwiched between two orthogonal polarizers to produce a spatially varying delay. Alpha-barium borate $(\alpha \mathrm{BBO})$ crystals are used with a nominally $5 \mathrm{~mm} 15^{\circ}$ cut angle crystal in the lower divertor system and a $5.8 \mathrm{~mm} 30^{\circ}$ cut angle in the main-SOL system. Interference filters with a $2 \mathrm{~nm}$ FWHM transmission width are mounted in a remote-controlled filter wheel mounted on the front of the interferometer cell. The cell, lens arrangement, filter wheel, and camera are rigidly fixed to an optical rail to minimize the movement of the crystal and lens relative to the camera. A photo of the diagnostic setup is shown in Fig. 2. To actively stabilize the crystal temperature, a series of resistive heating elements are adhered to the interferometer housing that are themselves surrounded by foam insulation. PID control is used to heat the crystal and aluminum mounting blocks to $33{ }^{\circ} \mathrm{C}$. The PID control thermistor for this heater setup is adhered to the top of the crystal with thermal epoxy. The interferometer cell and camera are inside an air-cooled shield box constructed from borated polyethylene panels to provide neutron attenuation, lead bricks to provide x-ray shielding, and stress annealed iron for magnetic shielding.

Absolute velocity calibration is achieved using a model of the interferometer and a reference image of a well-known spectral source illuminating an integration sphere inserted into the CIS field-of-view. This calibration procedure has been outlined previously where an optimized system demonstrated accuracy to $0.2 \mathrm{~km} / \mathrm{s}$ over a $3.5 \mathrm{~nm}$ extrapolation range. ${ }^{13} \mathrm{In}$ the lower divertor system, a pneumatic mirror is inserted into the optical train to bring the integration sphere into view. In the main-SOL system, the integration sphere itself moves into the optical train. In both cases, the light directed into the sphere can either be light from a tunable diode laser or a spectral lamp. Where the laser is used for reference images, a HighFinesse WS7 wavemeter provides a measurement of the laser wavelength with an accuracy of $0.04 \mathrm{pm}$ at $465 \mathrm{~nm}$. For the HeII measurements described here, a zinc spectral lamp was used for the calibration images. These lamps have a prominent spectral line at $468.014 \mathrm{~nm}$, about $0.55 \mathrm{~nm}$ from the measured HeII line at $468.571 \mathrm{~nm}$. While this extrapolation difference is

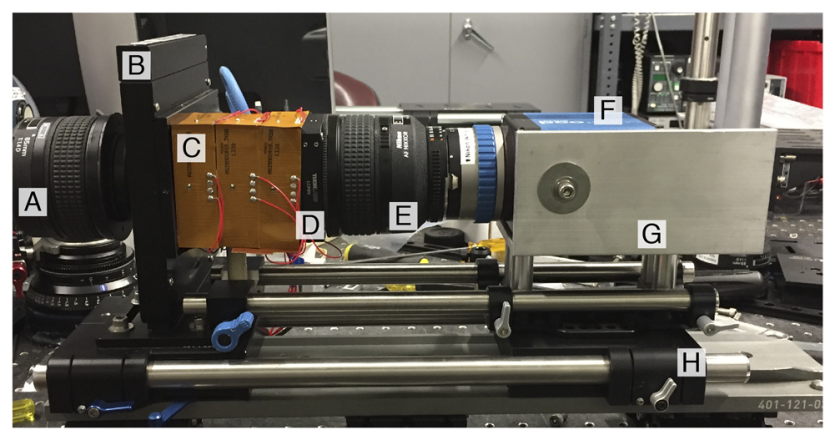

FIG. 2. Photograph of the main-SOL instrument before mounting in the shield box. Displayed components include A-Front $85 \mathrm{~mm}$ lens (collimator); $\mathrm{B}$ - filter wheel; $\mathrm{C}$-resistive heater elements surrounding interferometer cell; D-rear of interferometer cell; E-rear 85 mm lens; F-camera; G-camera mounting plate; and $\mathrm{H}$-rigid optical rail. 
non-optimal, it allowed switching between CIII and HeII configurations faster than could be achieved by retuning the diode laser. A LabView control system is used to co-ordinate the cameras, filter wheels, integration sphere translation, lamps, and wavemeter for the calibration sequence, the results of which are stored in local and remote MDSplus trees and a S3 object store system.

\section{ENVIRONMENTAL EFFECTS}

While one of the advantages of CIS is its high sensitivity, this sensitivity also makes it vulnerable to environmental influences. These environmental influences include thermal drift of the crystal, stress-induced birefringence, vibration, and magnetic field effects. These amount to both direct changes to the crystal that alter the interferometric delay, as well as changes in the relationship between the crystal and the camera that alter the interferogram's position on the CCD and therefore manifest as a phase-change. The influence of these effects on the DIII-D CIS systems is evaluated using a "calibration configuration" whereby the integration sphere used for post-shot calibration is placed into the CIS field-of-view during plasma operations. The wavelength of the light is well known, either from NIST wavelength standards for spectral lamps or using the wavemeter for the diode laser. The "velocity" of this source can then be calculated using the technique used for ascertaining plasma velocities; in an ideal scenario, the Doppler shift of the light source will always be zero. Any deviation from zero during the course of a plasma shot or over the course of a day can be used to evaluate the impact of environmental effects in a quantitative way and provide experimental uncertainty values.

\section{A. Thermal drift}

A birefringent crystal's refractive indices are a function of the crystal temperature and so thermal drifts cause phase shifts that will be incorrectly interpreted as Doppler shifts. Where this temperature drift occurs between the plasma measurement and the calibration image measurement, it causes a systematic velocity offset across the velocity image. Novel approaches have been presented to solve this problem including passive temperature stabilization by combining birefringent crystals with opposing thermal expansion and thermo-optic coefficients. ${ }^{17}$ The approach taken on DIII-D is to combine modest active temperature stabilization with fast calibration measurements under the premise that only a significant change in temperature between the plasma measurement and the calibration measurement is important. Active temperature stabilization is achieved using the PID control of resistive heaters adhered to the exterior of the interferometer cell. A layer of foam insulation separates this region from the rest of the shield box which is air-cooled to protect the cameras from over-heating. Typical temperature drifts for both systems over a DIII-D run day are shown in Fig. 3. These measurements were made by tracking the phase-shift between each post-shot laser calibration image and calculating the associated velocity drift. Over a $10 \mathrm{~h}$ span, the main-SOL system experienced a $29 \mathrm{~km} / \mathrm{s}$ drift with a maximum gradient of $9.7(\mathrm{~km} / \mathrm{s}) / \mathrm{h}$, whereas the lower divertor experienced a $8 \mathrm{~km} / \mathrm{s}$ drift with a maximum gradient

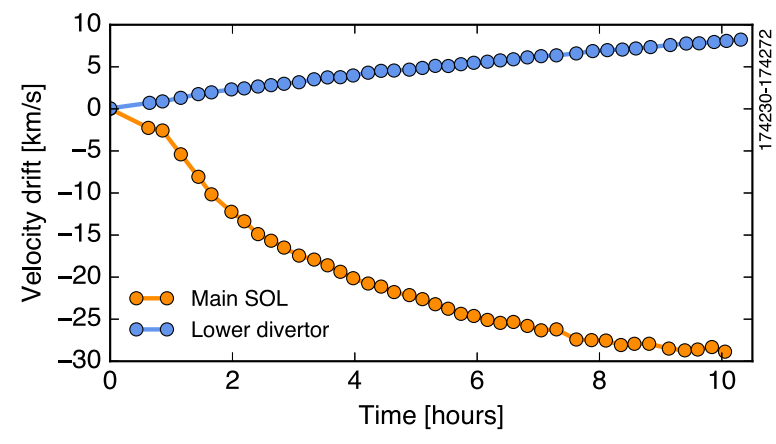

FIG. 3. Velocity drift over the course of a DIII-D run day primarily due to crystal temperature changes.

of $1.5(\mathrm{~km} / \mathrm{s}) / \mathrm{h}$. The faster rate of change in the main-SOL system is due to heating from a FLIR infra-red camera diagnostic that shares the shield box. Better temperature stability has been observed in benchtop systems where a stability of $0.08(\mathrm{~km} / \mathrm{s}) / \mathrm{h}$ over $5 \mathrm{~h}$ has been achieved. ${ }^{19}$ However, the degree of engineering required to achieve this stability is not a practical solution for diagnostics installed on the tokamak where space is limited due to radiation shielding requirements. To mitigate the effect of the residual thermal drift, calibration data by the automated acquisition system are acquired quickly after every shot. The time between plasma shot and calibration data is around $20 \mathrm{~s}$ for the main-SOL system and about $2 \mathrm{~min}$ for the lower divertor. The difference in response times between the two systems is due to the different memoryhandling capability of the pco.edge and Phantom cameras. The combination of fast calibration and active temperature stabilization reduces the error due to thermal drifts to be less than $0.06 \mathrm{~km} / \mathrm{s}$ in the main-SOL system and $0.05 \mathrm{~km} / \mathrm{s}$ in the lower divertor.

\section{B. Magnetic field}

While not yet fully understood, magnetic fields have been observed to impact CIS systems both due to field-induced mechanical movement and the direct influence of the magnetic field on the birefringent crystal itself. This effect was originally observed in an early iteration of the main-SOL system whereby a phase shift of up to 0.01 fringe $(\sim 3 \mathrm{~km} / \mathrm{s})$ occurred during a DIII-D plasma shot where the CIS diagnostic was in a "calibration configuration" observing HeNe laser light. This configuration has been used extensively to test the resilience of the diagnostic to environmental influence. The calibration source is placed into the camera's field of view during a plasma shot, and the phase shift is monitored throughout the discharge; there is no view of the plasma. Provided the wavelength of the light source is stable, the calculated Doppler velocity for this setup should be zero across the image and for all times. Any deviation from zero represents a diagnostic error that can be used for quantifying uncertainty of plasma measurements.

To separate out contributions from individual magnetic field coils and eliminate the possibility of stray-light contamination, a sequence of coils were individually energized comprising the strongest and closest coil sets to the main-SOL diagnostic location. A schematic of the main-SOL CIS location in reference to the DIII-D magnetic field coils is shown 


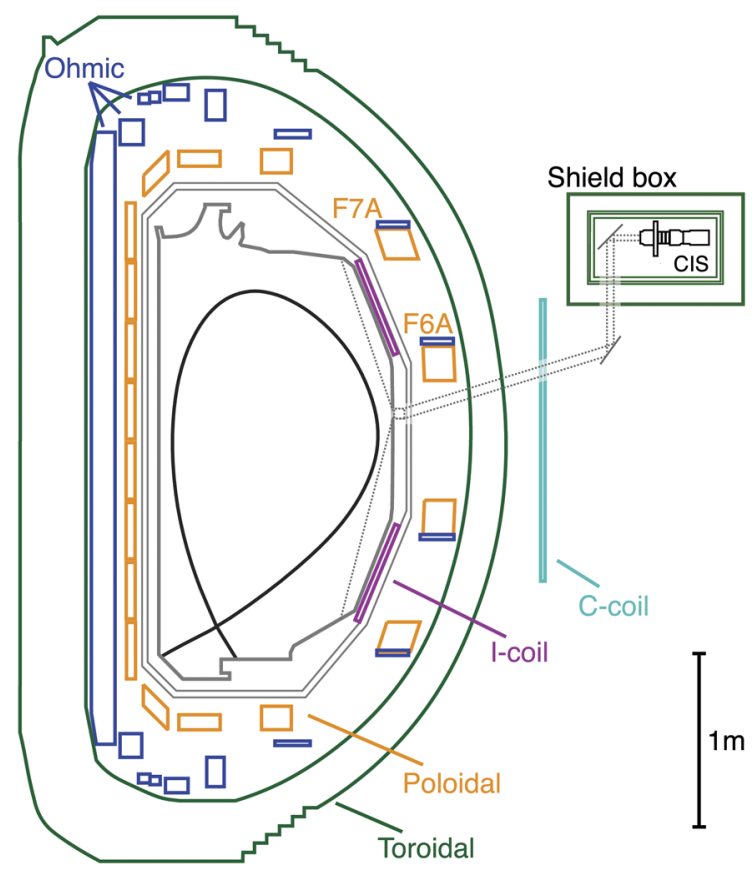

FIG. 4. Cross section of DIII-D showing the position of the main-SOL CIS system alongside the DIII-D magnetic field coils. Approximate length scale shown.

in Fig. 4. Coil currents were chosen to be close to the nominal values used during routine plasma operation, although exact values depend on the magnetic equilibrium shape and 3D field configuration for any given discharge. Maximum coil currents used were $109 \mathrm{kA}$ in the ohmic coil (Ecoil), $4 \mathrm{kA}$ in two of the shaping coils (F6A and F7A), $1.6 \mathrm{kA}$ in an external 3D coil centered at a torodial angle of $79^{\circ}$ (C79), and $3.1 \mathrm{kA}$ in a $3 \mathrm{D}$ coil internal to the DIII-D vacuum vessel centered at a toroidal angle of $90^{\circ}$ (IU90). The results of this test are shown in Fig. 5 where it can be seen that the ohmic coil and F6A shaping coil elicit the greatest phase response. A single-axis
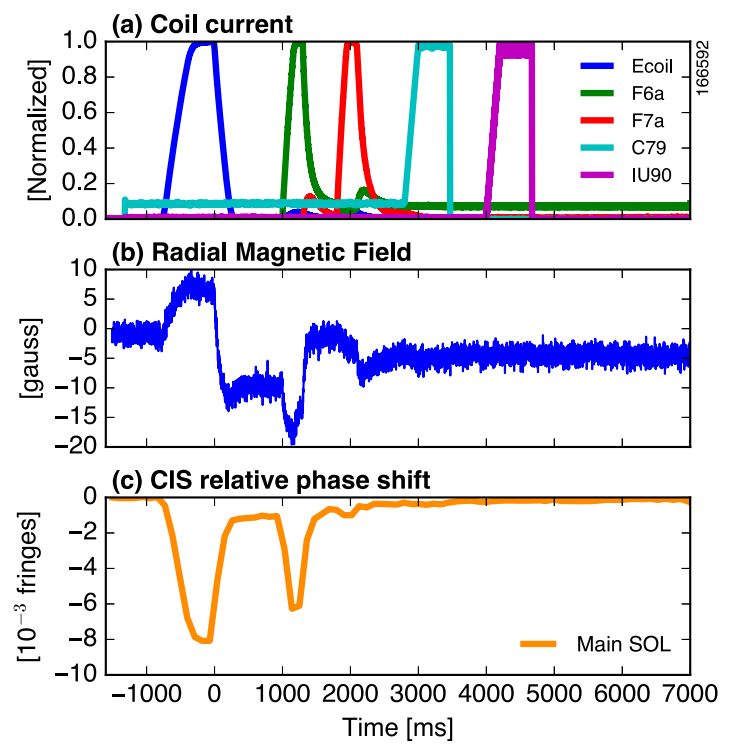

FIG. 5. A sequence of current pulses applied to individual DIII-D magnetic coils to test the magnetic field dependence of CIS. (a) Normalized current in each coil. (b) Radial magnetic field strength measured in the SOL system shield box. (c) Phase change measured for a HeNe laser with stable wavelength. gauss meter placed near the interferometer cell and aligned to measure radial fields (along the CIS axis) showed that phase changes up to 0.008 fringes resulted from radial fields up to $-20 \mathrm{G}$ but that the phase response was not linear with radial field. This phase shift corresponds to about $1.5 \mathrm{~km} / \mathrm{s}$ velocity shift for CIII at $465 \mathrm{~nm}$. Furthermore, no linear superposition of coil currents multiplied by individual coefficients could reproduce the time history during both the early and late portions of a DIII-D plasma shot indicating that the magnetic field generated by the plasma current (1 MA loop) is significant.

Increasing the magnetic shielding around CIS cell only reduced the phase-shift during plasma operation by about $30 \%$. A revised mounting system that held the CIS cell more rigidly and fixed the CIS cell, camera, and lenses to the shield box generated a factor of six improvement. This indicates that the magnetic field was inducing mechanical movement in a component of the system that produced the phase shift. However, benchtop testing with permanent magnets confirmed that the magnetic field can directly impact the crystal. In this test, a permanent magnet was placed near the crystal while the phase of a spectral lamp was being monitored. When the magnetic was positioned $4 \mathrm{~cm}$ above the birefringent crystal such that the field at the crystal was about $300 \mathrm{G}$, a phase shift of 0.02 fringes was detected. This phase shift was constant while the crystal remained stationary. Using a stationary permanent magnet isolates the magnetic field itself as being able to generate a phase shift, as opposed to an electric field or eddy currents. These other effects may still be influencing the crystal in the more complex tokamak environment. Rotating the magnet pole-wise or azimuthally did not affect the sign of the phase shift indicating that the effect of the magnetic field on the crystal is not as straight forward as a simple Faraday rotation. This is most likely due to the inherent non-linearity of birefringent crystals; Faraday rotation in birefringent crystals oscillates about zero as a function of the path length. ${ }^{20}$ Furthermore, the cut angle of the crystals used in the CIS setups described here is non-orthogonal to the light's propagation direction and so the magnetic field angle will be important. Three-axis Helmholtz characterization of these crystals is required to fully understand the effect.

In the current main-SOL design, the intra-shot drift has a magnitude of up to $0.5 \mathrm{~km} / \mathrm{s}$, as seen in Fig. 6. While physically remounting the diagnostic assembly reduced the impact of magnetic fields to an acceptable value, it is clear that magnetic fields can have a significant effect on a CIS instrument and

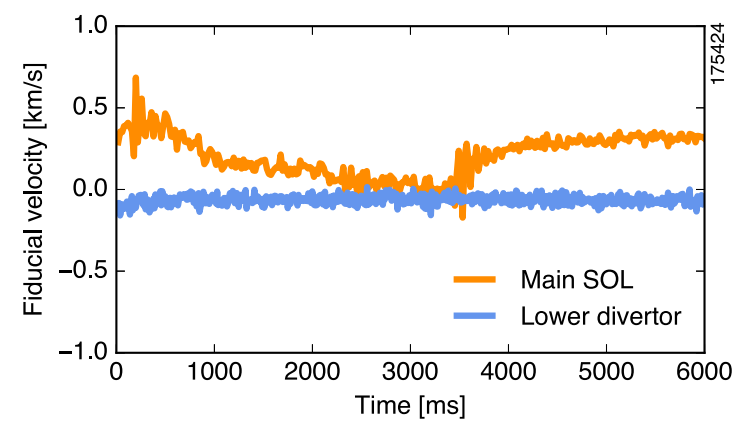

FIG. 6. Average effective velocity of laser fiducial during a DIII-D discharge for both lower divertor (blue) and SOL (orange) systems. 
must be considered carefully in situations where the diagnostic is exposed to magnetic fields.

\section{Vibration}

A number of prominent sources of vibration exist in the vicinity of the DIII-D vessel due to the routine operation of pumps and fans, and from plasma disruptions and Edge Localized Modes (ELMs) that can shake the tokamak. When these vibrations rigidly move the entire instrument, they only alter the spatial alignment which can be corrected using in-vessel fiducials on a frame-by-frame basis. These changes tend to be small linear deviations $(<10$ pixels) or rotations $\left(<5^{\circ}\right)$ applied to the in-vessel calibrations completed during infrequent machine-openings. However, changing the position of the crystal with respect to the camera, or altering the backward facing lens position, alters the registration of the fringes on the detector and so appears as a spurious phase shift. To combat these effects, a rigid mounting system was developed using cinema-grade optical rails. This system creates a common mounting platform for the camera, interferometer cell, imaging lenses, and filter wheel. The performance of this system can be measured using the same dataset used to quantify the effect of magnetic fields, shown in Fig. 5. High frequency (frame-to-frame) oscillations in the average velocity across the image were observed in both the mainSOL and lower divertor systems. For both the lower divertor and main-SOL systems, high-frequency oscillations display a peak-to-peak magnitude of around $0.05 \mathrm{~km} / \mathrm{s}$. These results indicate that the mounting system adequately shields against vibration noise.

Since the phase-shifts due to vibration and those due to magnetic fields described above tend to be systematic phase shifts applied across the image, they could be easily countered using intra-shot calibration. This could be achieved using very stable light source, a laser, for example, that illuminates an otherwise unused part of the CCD. This light source would provide information about systematic shifts that can then be removed in post-processing.

\section{DIAGNOSTIC COMPARISON}

Direct comparison of CIS with an independent diagnostic technique provides a stringent test of the CIS implementation. One of the weaknesses of the CIS technique is the use of an interference filter that passes a discrete band of spectral emission. This requires assumptions to be made about the quality and consistency of the line shape that can lead to significant errors; spectral impurities, or an asymmetric change in the line shape, will appear as Doppler shifts and are impossible to detect with CIS alone. Traditional spectroscopy does not suffer from this limitation and so is well suited for providing an independent test of CIS operation. Additionally, using a spectroscopic velocity measurement ensures that the measured velocity is of the same ion population being sampled with CIS. This avoids the need to rely on assumptions about ion entrainment or charge state concentrations as would be the case with a probe-based comparison.

There are three main areas of concern for CIS that are addressed with a traditional spectroscopy comparison: spectral impurities passing through the interference filter bandpass; thin films on in-vessel optics causing the preferential throughput of only right-handed or left-handed circularly polarized sates from Zeeman-split spectra; and geometric differences in light collection optics between the calibration and tokamak light sources that invalidates absolute calibration from ex-vessel light sources. In each case, attempts have been made to quantify the degree of influence that the effect might be having on velocity measurements. These included spectrometer surveys accumulating a wide range of discharges; in-vessel polarization tests to identify changes in polarization extinction thresholds due to in-vessel optics; and the construction of full-size replica optics for testing. While in each case these tests gave positive indications, the challenging tokamak viewing geometry and the nature of in-vessel optics make it difficult to draw firm conclusions. The more rigorous test is the comparison with traditional spectroscopy that is presented here.

\section{A. Traditional spectroscopy setup}

Spectroscopic ion velocity measurements have previously been made on DIII-D using the high resolution Multi-Chordal Divertor Spectrometer (MDS). ${ }^{8,21}$ With this spectrometer, both vertical and mostly-tangential viewchords are simultaneously measured using a Princeton Instruments PI-MAX 4 intensified camera. The spectrometer itself is a McPherson $1.3 \mathrm{~m}$ Czerny-Turner fitted with a $1200 \mathrm{l} / \mathrm{mm}$ grating with a dispersion of about $0.07 \AA /$ pixel. The wavelength offset between viewchords is calculated using zinc and cadmium fiducial lines at $468.014 \mathrm{~nm}$ and $465.112 \mathrm{~nm}$, respectively. The magnitude of this correction ranged up to $0.08 \AA$, equivalent to a Doppler shift of about $5 \mathrm{~km} / \mathrm{s}$ at these wavelengths.

In previous work, the method for calculating the velocity for a given time slice used an iterative fitting procedure that takes into account the local Zeeman splitting, multiple ion populations, and the viewchord geometry. The Zeeman splitting aspect of the fit was used to localize the emission in combination with magnetic field information from the magnetic equilibrium reconstruction code EFIT. ${ }^{22}$ The velocity fiducial was taken from one of the vertical chords, ideally the one that passes near to the x-point where the line-averaged velocity can assumed to be zero due to the view angle being mostly perpendicular to the magnetic field. Several improvements to this method have been implemented for the present work. First, one of the spectrometer viewchords was connected to an integration sphere with the aforementioned $\mathrm{Zn}$ and $\mathrm{Cd}$ spectral lamps. This provided an intra-shot wavelength and dispersion reference to ensure a high quality wavelength fiducial specific to each plasma shot and one that does not require any plasma assumptions. However, it is worth noting that for the lower single null diverted plasmas examined here, the velocity of the lower-viewing chords passing near the X-point had a zero Doppler shift to within the experimental accuracy. This confirms the previously used approach.

The second improvement was the removal of Zeeman localization in favor of using tomographically inverted emissivity profiles to determine where the emission is originating from. The CIS emissivity profiles were used for 


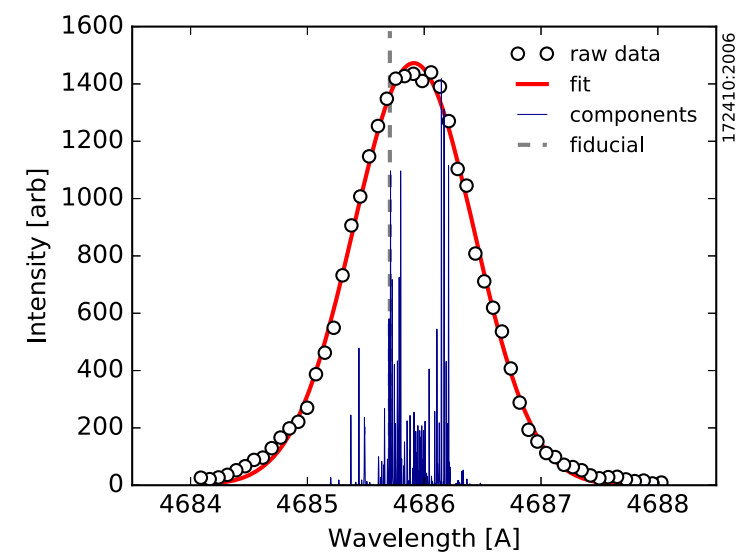

FIG. 7. Heir spectra observed using MDS along a tangential view of the divertor. Measured spectra (open circles) are shown alongside the fitted line shape (red line) comprised of 146 Zeeman components (blue lines) calculated at a magnetic field of $2 \mathrm{~T}$ and viewing angle of $148^{\circ}$. The Doppler shift for this case is the wavelength difference between the peak of the fitted spectra and the zero-velocity fiducial (vertical dashed line) corresponding to a velocity of $12.7 \mathrm{~km} / \mathrm{s}$.

this purpose. From this localization, the local magnetic field can be obtained from the EFIT and then used for the calculation of the Zeeman splitting. An exact calculation of the Zeeman components and their relative intensity is determined using the method outlined by Isler et al. in Appendix B of Ref. 23. This method calculates a set of relative intensities and wavelength positions for a given electronic transition for arbitrary viewing angle and arbitrary magnetic field strength without needing to make high- or low-field approximations. A typical HeII line shape plotted alongside its constituent 146 Zeeman components is shown in Fig. 7. The instrumental broadening was unusually large for these cases due to a recent change in spectrometer focus which results in an instrument-dominated spectral width, however this does not impact the Doppler shift calculation. In the low temperature divertor region, it is expected that broadening to Zeeman splitting is the next largest contributor to the width, followed by temperature broadening. The uncertainty in velocities determined with this method is approximated to be \pm 0.5 pixels based on the determination of fiducials and the need for track-to-track calibration which are the two greatest sources of error. This corresponds to $\pm 2.5 \mathrm{~km} / \mathrm{s}$ which is mostly systematic.

\section{B. Velocity comparison}

To compare CIS with traditional spectroscopy, measurements were made in a pure helium plasma with $0.98 \mathrm{MA}$ plasma current, a toroidal field of $1.68 \mathrm{~T}$ (ion $\mathbf{B} \times \nabla \mathbf{B}$ down), the plasma shape shown in Fig. 4, and about 1.5 MW of electron cyclotron heating $(\mathrm{ECH})$ to generate a low confinement (L-mode) plasma without ELMs. Density was ramped over the course of the discharge using He gas puffing that generates a change in detachment conditions in the divertor. The density trajectory is shown in Fig. 8 alongside measurements of peak heat flux measured using an infrared camera system and electron temperature measured near the divertor surface at the outer target measured with Thomson scattering. Heat flux and electron temperature measurements show the transition from
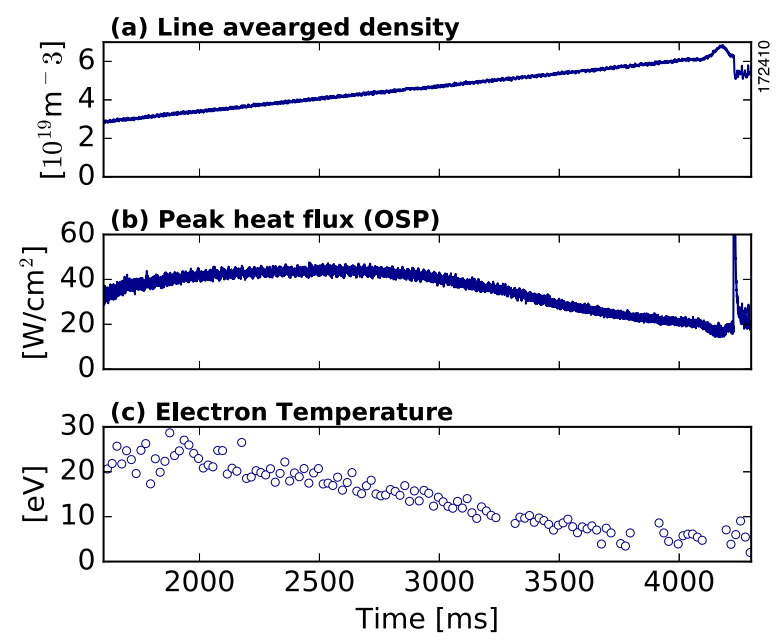

FIG. 8. Discharge evolution of a pure helium DIII-D discharge. Electron density is ramped using He gas puffing (a) causing a reduction in peak heat flux (b) and electron temperature (c) measured at the outer divertor target plate signifying the onset of a partially detached state.

well-attached to partially detached conditions. While there are several quasi-tangential viewchords available, the "T3" MDS viewchord was chosen for ease of interpretation. The trajectory of this viewchord can be seen in Fig. 9. It only passes through a single divertor leg and so avoids the complication of accounting for two separate ion populations traveling in opposite directions as would be the case if both inner and outer divertor legs were sampled.

Tomographic inversions of 300 CIS time slices were produced using 300 iterations for both emissivity and velocity whereby a $2 \mathrm{D}$ profile of $\mathrm{He}^{+}$velocity parallel to the magnetic field is calculated. Full details on the inversion process have been published elsewhere. ${ }^{24}$ Obvious reflections are masked using a user-defined mask. Examples of the inverted emissivity and velocity profiles are shown in Fig. 9 for an attached case and Fig. 10 for a partially detached case. To remove the impact of low-signal inversion artifacts, both the emissivity and velocity are masked using an emissivity threshold of 5\% of the maximum emissivity in each time slice. This masking has been previously shown to have only a small impact on the sum of the mean squared error. ${ }^{14}$

The partial detachment onset causes the region of HeII emissivity to become less localized and to rise up the divertor leg as the electron temperature falls. One impact of this change in emissivity location is that the position of reflections also changes location. While reflections from DIII-D's carbon walls are substantially smaller than those of a metal-walled machine, during partially detached conditions, reflections may account for as much as $10 \%$ of the emissivity and so impact the tomographic inversion process. The emissivity observed along the shelf tiles in Fig. 10, for example, at $Z=-1.2 \mathrm{~m}$ and $R=1.5-1.7 \mathrm{~m}$, may be indicative of this problem.

Whereas the tomographic inversion of CIS data calculates ion velocity along parallel field lines, MDS makes line-ofsight velocity measurements that are specific to the particular viewchord geometry. To enable a direct comparison between the two diagnostics, the CIS velocity solution was interpolated onto the MDS viewchord using an emissivity-weighted 

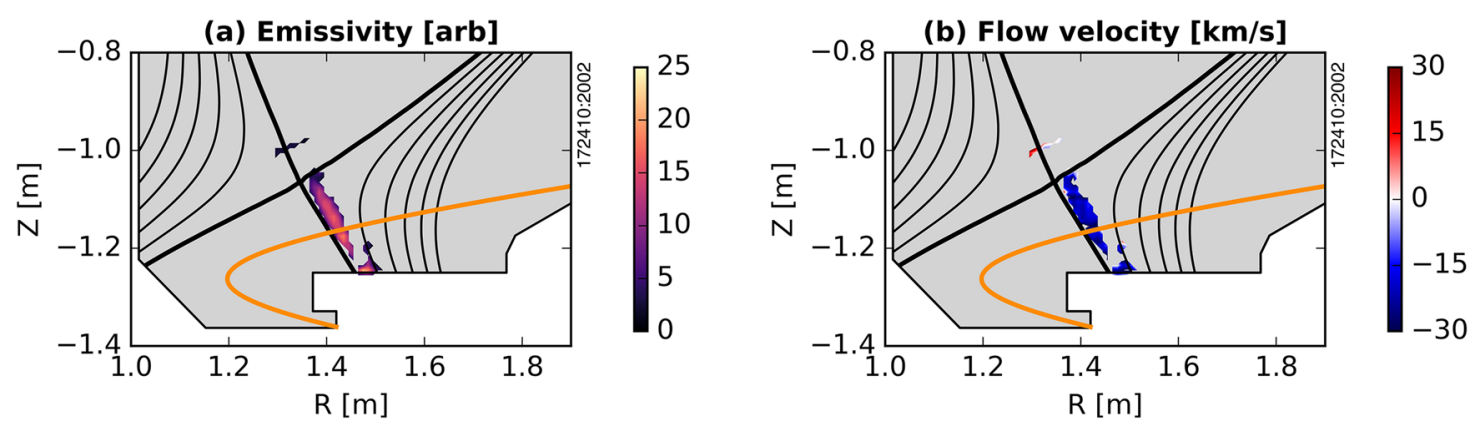

FIG. 9. (a) CIS emissivity and (b) velocity measurements for plasma conditions with an attached outer divertor leg and overlaid with the MDS T3 viewchord (orange line).
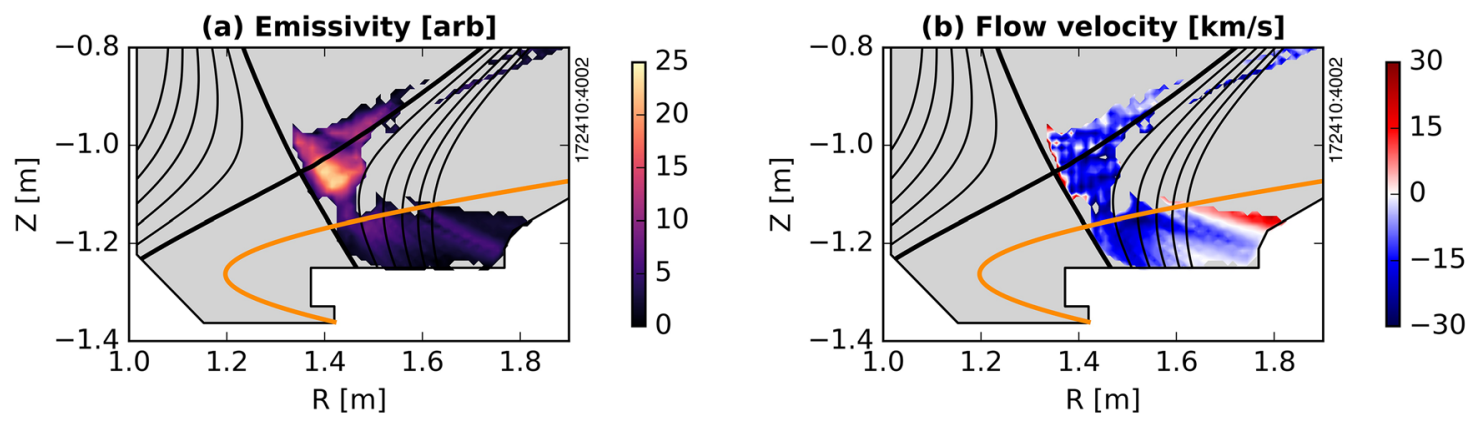

FIG. 10. (a) CIS emissivity and (b) velocity measurements for plasma conditions with a partially detached outer divertor leg and overlaid with the MDS T3 viewchord (orange line).

average. Taking the dot product between the viewchord geometry at each spatial location and the local magnetic field direction gives the line-of-sight projection of the parallel velocity. An underlying source of error in this approach is the spatial alignment of the viewchord; however, the magnetic field and flow velocity are not expected to vary significantly under these conditions.

The comparison between CIS and traditional spectroscopy velocities is shown in Fig. 11. Quantitative agreement during attached conditions (2000-3000 ms) is very good. Once the plasma begins to detach, both CIS and MDS display a slowing velocity. This slowing is not the same trend as has been observed on DIII-D previously $;{ }^{8}$ however, discharge conditions vary substantially from previous work and so the difference remains a topic for further investigation. The velocity slowing observed by CIS occurs about $550 \mathrm{~ms}$ before the

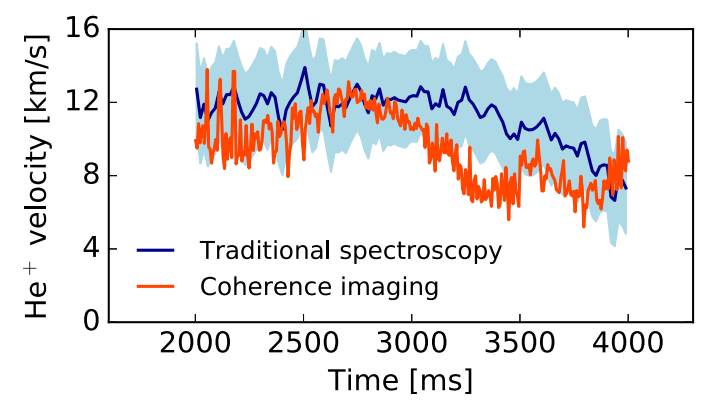

FIG. 11. Line-of-sight helium ion velocities along the T3 MDS viewchord as measured by MDS (purple) and calculated using CIS measurements (orange) for the discharge described in Fig. 8. Error bars for the MDS measurements are shown in as a light-blue band. equivalent MDS observation that may indicate an error with the vertical alignment of the T3 chord. During this time, the ionization front and associated emissivity are rising up through the MDS viewchord. Agreement is not as good during an intermediate phase between $3200 \mathrm{~ms}$ and $3500 \mathrm{~ms}$ before improving again. However, this improvement occurs during the same time period that reflections become more problematic indicating that while line-of-sight velocities are in good agreement, the localization of these velocities may be erroneous. This issue highlights the reason that tomographic inversions of velocity are substantially more difficult than emissivity inversions. Whereas emissivity can only take positive values, velocity can be positive or negative meaning that it is difficult to distinguish between a chord viewing a zero velocity population and a chord viewing two very fast populations travelling in opposite directions but whose mean velocity is zero.

While this diagnostic comparison does not prove that the issues of thin films, geometric differences, and spectral impurities are not present in the CIS diagnostic, if these effects were large, they would manifest as a significant discrepancy between velocities determined by CIS and traditional spectroscopy. The discovery that reflections can be problematic for detached helium plasmas even in a carbon machine suggests that work to include the surface reflectivity in tomographic reconstructions could further improve the accuracy of CIS on DIII-D.

\section{SUMMARY}

A series of experiments and diagnostic tests have been undertaken to demonstrate the accuracy and reliability of 
CIS velocity measurements on DIII-D with the target of attaining $1 \mathrm{~km} / \mathrm{s}$ uncertainty. By combining active temperature control and automated calibration between shots, the effect of thermal drift was shown to be less than $0.06 \mathrm{~km} / \mathrm{s}$. Intra-shot drift due to the nearby strong magnetic fields was reduced to $0.5 \mathrm{~km} / \mathrm{s}$ in the main-SOL system and less than $0.1 \mathrm{~km} / \mathrm{s}$ in the lower divertor setup. A novel mounting system to rigidly constrain optical components reduced vibration noise to less than $0.05 \mathrm{~km} / \mathrm{s}$. Comparison with traditional spectroscopy in the lower divertor showed good agreement, particularly where reflective surfaces did not impact tomographic reconstructions. Future work will focus on reducing demodulation noise and tomographic artifacts related to these reflections.

\section{ACKNOWLEDGMENTS}

This material is based upon work supported by the U.S. Department of Energy, Office of Science, Office of Fusion Energy Sciences, using the DIII-D National Fusion Facility, a DOE Office of Science user facility, under Award Nos. DEFC02-04ER54698 (DIII-D), DE-AC52-07NA27344 (LLNL), and DE-AC05-00OR22725 (ORNL). DIII-D data shown in this paper can be obtained in digital format by following the links at https://fusion.gat.com/global/D3D_DMP.

This report was prepared as an account of work sponsored by an agency of the United States Government. Neither the United States Government nor any agency thereof, nor any of their employees, makes any warranty, express or implied, or assumes any legal liability or responsibility for the accuracy, completeness, or usefulness of any information, apparatus, product, or process disclosed, or represents that its use would not infringe privately owned rights. Reference herein to any specific commercial product, process, or service by trade name, trademark, manufacturer, or otherwise, does not necessarily constitute or imply its endorsement, recommendation, or favoring by the United States Government or any agency thereof. The views and opinions of authors expressed herein do not necessarily state or reflect those of the United States Government or any agency thereof.

${ }^{1}$ M. Groth, J. Boedo, N. Brooks, R. Isler, A. Leonard, G. Porter, J. Watkins, W. West, B. D. Bray, M. Fenstermacher et al., "Effect of cross-field drifts on flows in the main scrape-off-layer of DIII-D L-mode plasmas," Nucl. Fusion 49, 115002 (2009).

${ }^{2}$ S. Erents, R. Pitts, W. Fundamenski, J. Gunn, and G. Matthews, "A comparison of experimental measurements and code results to determine flows in the JET SOL," Plasma Phys. Controlled Fusion 46, 1757 (2004).

${ }^{3}$ R. M. Churchill, J. M. Canik, C. Chang, R. Hager, A. W. Leonard, R. Maingi, R. Nazikian, and D. P. Stotler, "Kinetic simulations of scrape-off layer physics in the DIII-D tokamak," Nucl. Mater. Energy 12, 978-983 (2017). ${ }^{4} \mathrm{~N}$. Asakura, ITPA SOL, and Divertor Topical Group, "Understanding the SOL flow in L-mode plasma on divertor tokamaks, and its influence on the plasma transport," J. Nucl. Mater. 363, 41-51 (2007).
${ }^{5}$ N. Smick, B. LaBombard, and I. Hutchinson, "Transport and drift-driven plasma flow components in the Alcator C-Mod boundary plasma," Nucl. Fusion 53, 023001 (2013).

${ }^{6}$ J. Boedo, G. Porter, M. Schaffer, R. Lehmer, R. Moyer, J. Watkins, T. Evans, C. Lasnier, A. Leonard, and S. Allen, "Flow reversal, convection, and modeling in the DIII-D divertor," Phys. Plasmas 5, 4305-4310 (1998).

${ }^{7}$ J. Boedo, E. Belli, E. Hollmann, W. Solomon, D. Rudakov, J. Watkins, R. Prater, J. Candy, R. Groebner, K. Burrell et al., "Poloidally and radially resolved parallel D+ velocity measurements in the DIII-D boundary and comparison to neoclassical computations," Phys. Plasmas 18, 032510 (2011).

${ }^{8}$ R. Isler, N. Brooks, W. West, A. Leonard, G. McKee, and G. Porter, "Spectroscopic analysis of normal and reversed ion flows in the DIII-D divertor," Phys. Plasmas 6, 541-549 (1999).

${ }^{9}$ J. Howard, C. Michael, F. Glass, and A. Danielsson, "Time-resolved twodimensional plasma spectroscopy using coherence-imaging techniques," Plasma Phys. Controlled Fusion 45, 1143 (2003).

${ }^{10}$ J. Howard, A. Diallo, M. Creese, S. Allen, R. Ellis, W. Meyer, M. Fenstermacher, G. Porter, N. Brooks, M. Van Zeeland et al., "Coherence imaging of flows in the DIII-D divertor," Contrib. Plasma Phys. 51, 194-200 (2011).

${ }^{11}$ J. Howard, A. Diallo, M. Creese, B. Blackwell, S. Allen, R. Ellis, G. Porter, W. Meyer, M. Fenstermacher, N. Brooks et al., "Doppler coherence imaging and tomography of flows in tokamak plasmas (invited)," Rev. Sci. Instrum. 81, 10E528 (2010).

${ }^{12}$ S. Silburn, J. Harrison, J. Howard, K. Gibson, H. Meyer, C. Michael, and R. Sharples, "Coherence imaging of scrape-off-layer and divertor impurity flows in the mega amp spherical tokamak (invited)," Rev. Sci. Instrum. 85, 11D703 (2014)

${ }^{13}$ C. Samuell, S. Allen, W. Meyer, and J. Howard, "Absolute calibration of Doppler coherence imaging velocity images," J. Instrum. 12, C08016 (2017).

${ }^{14}$ C. Samuell, G. Porter, W. Meyer, T. Rognlien, S. Allen, A. Briesemeister, A. Mclean, L. Zeng, A. Jaervinen, and J. Howard, "2D imaging of helium ion velocity in the DIII-D divertor," Phys. Plasmas 25, 056110 (2018).

${ }^{15} \mathrm{D}$. Gradic et al., "Absolutely calibrated doppler coherence imaging flow measurements in the divertor of a tokamak," in talk presented at 2nd European Conference on Plasma Diagnostics (ECPD 2017), 2017.

${ }^{16}$ C. Michael, J. Howard, and B. Blackwell, "The MOSS camera on H-1NF," Rev. Sci. Instrum. 72, 1034-1037 (2001).

${ }^{17}$ R. Lester, Y. Zhai, C. Corr, and J. Howard, "Coherence imaging for ion temperature and flow measurements in a low-temperature helicon plasma source," Plasma Sources Sci. Technol. 25, 015025 (2016).

${ }^{18}$ C. J. Lasnier, S. L. Allen, R. E. Ellis, M. E. Fenstermacher, A. G. McLean, W. H. Meyer, K. Morris, L. Seppala, K. Crabtree, and M. A. Van Zeeland, "Wide-angle ITER-prototype tangential infrared and visible viewing system for DIII-D,” Rev. Sci. Instrum. 85, 11D855 (2014).

${ }^{19}$ S. Allen, C. Samuell, W. Meyer, and J. Howard, "Laser calibration of the DIII-D coherence imaging system," Rev. Sci. Instrum. (unpublished).

${ }^{20}$ A. Kurtzig, "Faraday rotation in birefringent crystals," J. Appl. Phys. 42, 3494-3498 (1971).

${ }^{21}$ R. Isler, N. Brooks, W. West, and G. Porter, "Carbon flows in attached divertor plasmas," Phys. Plasmas 6, 1837-1842 (1999).

${ }^{22}$ J. Weaver, B. Welch, H. Griem, J. Terry, B. Lipschultz, C. Pitcher, S. Wolfe, D. Pappas, and C. Boswell, "Localization of emission through interpretation of observed Zeeman pattern,” Rev. Sci. Instrum. 71, 1664-1670 (2000).

${ }^{23}$ R. Isler, R. Wood, C. Klepper, N. Brooks, M. Fenstermacher, and A. Leonard, "Spectroscopic characterization of the DIII-D divertor," Phys. Plasmas 4, 355-368 (1997).

${ }^{24}$ W. Meyer, S. Allen, C. Samuell, and M. Fentermacher, "Tomographic analysis of tangential viewing cameras," Rev. Sci. Instrum. (unpublished).

${ }^{25}$ J. Chung, R. König, J. Howard, M. Otte, and T. Klinger, "Time resolved coherence-imaging spectrometer on WEGA stellarator," Plasma Phys. Control. Fusion 47(6), 919 (2005). 